\title{
CD10 is useful in demonstrating endometrial stroma at ectopic sites and in confirming a diagnosis of endometriosis
}

\author{
V P Sumathi, W G McCluggage
}

J Clin Pathol 2002;55:391-392

\begin{abstract}
Aims: Recent studies have shown that CD10 is a useful immunohistochemical marker of normal endometrial stroma and of endometrial stromal neoplasms. The aim of this study was to investigate whether CDIO immunoreactivity is present in ectopic endometrial stroma and whether staining is of value in confirming a diagnosis of endometriosis.

Methods/Results: Twenty five cases of endometriosis were stained with a commercially available antibody against CD10. Endometrial stromal cells were positive in 22 of 25 cases. There was little or no staining of other tissues.

Conclusions: CD10 immunoreactivity is largely maintained in endometrial stromal cells located outside the uterus. Immunohistochemical staining with CD10 may be of value in confirming a diagnosis of endometriosis when there is morphological doubt.
\end{abstract}

A ntibodies against CD10 or common acute lymphoblastic leukaemia antigen (CALLA) are now available for routine immunohistochemistry on paraffin wax embedded tissues. CDI0 is expressed by haematopoietic neoplasms such as acute lymphoblastic leukaemia and follicular lymphomas, and antibodies are widely used in lymphoma and leukaemia panels. ${ }^{1}$ A recent study showed CDI0 expression in a limited number of non-haematopoietic tissues, including normal endometrial stromal cells and endometrial stromal sarcoma. ${ }^{2}$ This study identified CDI0 expression in renal tubular and glomerular cells, breast, and salivary gland myoepithelium, prostatic glandular epithelium, and pulmonary alveolar lining cells. Since that publication a small number of studies have shown that CDI0 is a useful immunohistochemical marker of endometrial stromal cells and is of diagnostic value in distinguishing endometrial stromal neoplasms from their histological mimics. ${ }^{34}$

"A recent study showed CD10 expression in a limited number of non-haematopoietic tissues, including normal endometrial stromal cells and endometrial stromal sarcoma"

Our small study aimed to assess the value of CD10 in identifying endometrial stromal cells at ectopic sites, thus establishing a definitive diagnosis of endometriosis.

\section{MATERIALS AND METHODS}

Table 1 summarises the cases of endometriosis used in our study. These were retrieved from the archives of the department of pathology, Royal Group of Hospitals Trust, Belfast. Twenty five cases of endometriosis at different sites were included. Diagnosis was made on haematoxylin and eosin stained sections and in most cases an unequivocal diagnosis of endometriosis was made. In three cases (one uterosacral ligament and two Caesarean section scars) a diagnosis of "suggestive of endometriosis" had been made.

A representative histological block from each of the 25 cases was chosen for immunohistochemistry. Sections were cut on to aminopropyltriethoxysilane treated slides (Sigma, Poole, Dorset, UK) and dried overnight at $37^{\circ} \mathrm{C}$. Immunolocalisation of CD10 (Clone 56C6; 7 pg/ml; Novocastra, Newcastle, UK) was performed using a peroxidase EnVision kit (Dako, Ely, UK). Diaminobenzidine was used as the chromogen and Harris's haematoxylin as the counterstain. For negative controls the primary antibody was omitted and replaced with immunoglobulin (IgGl, Dako) at an equivalent concentration. All sections were pretreated in $0.01 \mathrm{M}$ citrate buffer, $\mathrm{pH}$ 6.0, and microwaved at $850 \mathrm{~W}$ for 22 minutes before localisation. Positive controls consisted of normal tonsil. Positive staining was subjectively classified as weak, moderate, or strong.

\section{RESULTS}

Table 1 gives the immunohistochemical results. In all positive cases staining was cytoplasmic with no nuclear reactivity. Positivity was confined to endometrial stromal cells (fig l), with no staining of the glandular elements (except for minimal weak positivity in one case). Staining of the stromal cells was generally moderate to strong and diffuse, although there were occasional cases where staining was focal and of

Table 1 Cases included in the study together with results of immunohistochemistry

\begin{tabular}{llllll}
\hline Site & Number & Negative & Strong & Moderate & Weak \\
\hline Ovary & 8 & 1 & 7 & 0 & 0 \\
Caesarean section scar & 6 & 2 & 1 & 2 & 1 \\
Cervix & 4 & 0 & 1 & 2 & 1 \\
Uterosacral ligament & 3 & 0 & 1 & 2 & 0 \\
Large intestine & 2 & 0 & 0 & 2 & 0 \\
Umbilicus & 1 & 0 & 1 & 0 & 0 \\
Peritoneum & 1 & 0 & 1 & 0 & 0 \\
\hline
\end{tabular}




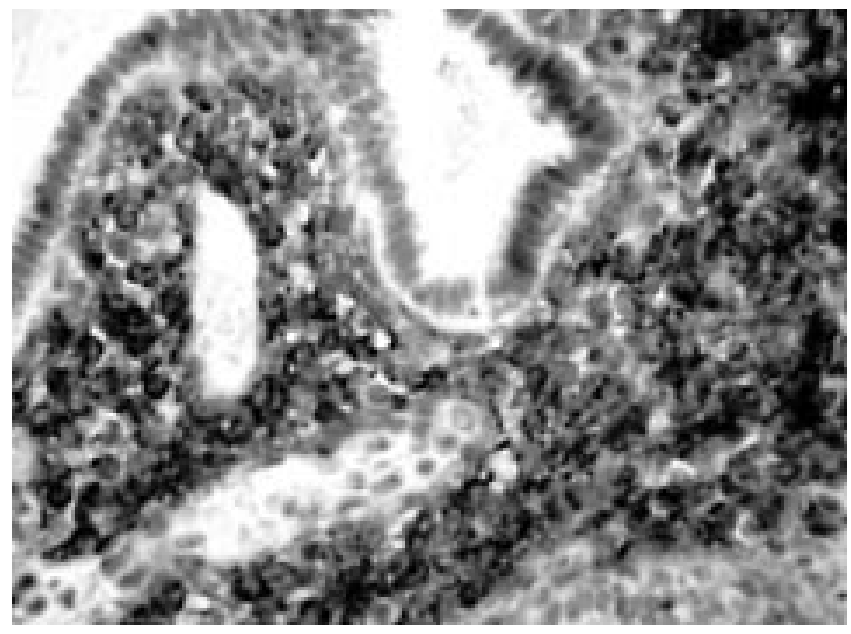

Figure 1 Strong immunohistochemical staining with CD 10 of stroma, but not glands, in a case of endometriosis.

weak intensity. Three cases were negative for CDI0 (two Caesarean section scars and one ovary). In one case of cervical endometriosis there was also focal weak staining of normal cervical stroma. There was no staining of other normal tissues.

In the positive controls there was staining of the germinal centre cells of normal tonsil. There was no staining of negative controls.

\section{DISCUSSION}

Recent studies have indicated that CDI0 is a very sensitive and diagnostically useful immunohistochemical marker of normal endometrial stroma and of endometrial stromal neoplasms, especially endometrial stromal nodule and low grade endometrial stromal sarcoma. ${ }^{34}$ In differentiating an endometrial stromal lesion from a cellular leiomyomatous tumour (the most common diagnostic dilemma), CDl0 should be used as

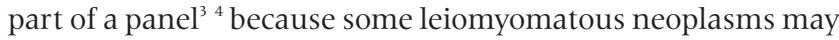
exhibit focal staining.

The results of our present study indicate that, where there is diagnostic doubt, immunohistochemical staining with CDI0 may be useful in identifying endometrial stroma (which otherwise lacks a characteristic immunohistochemical marker) and in confirming a diagnosis of endometriosis. Positivity was seen in 22 of 25 cases. Moreover, there were few or no problems with staining of other tissues, weak positive staining of normal cervical stroma being present in a single case. Endometriosis is defined as the presence of ectopic nonneoplastic endometrial tissue, usually glands and stroma, outside the uterus. This tissue in ectopic sites is almost invariably under the influence of ovarian hormones and therefore undergoes secondary changes such as bleeding and fibrosis. In those cases where endometrial-type glands and stroma are present, the histological diagnosis of endometriosis is often straightforward. However, especially in longstanding cases where the amount of endometrial stroma is sparse owing to fibrous obliteration, diagnosis becomes difficult. The distinction between non-specific fibrous stroma, ovarian stroma, and endometrial stroma can be difficult. In addition, in some cases, especially within the cervix, endometrial stroma may be present without glands, a condition known as stromal endometriosis. ${ }^{5}$ In these situations, examining multiple histological levels and performing CD10 immunohistochemistry

\section{Take home messages}

- In general, CD10 immunoreactivity is maintained in endometrial stromal cells located outside the uterus

- Immunohistochemical staining with CD10 may be useful in confirming a diagnosis of endometriosis when there is morphological doubt

may be extremely useful in confirming a diagnosis of endometriosis. Occasionally, especially with ovarian endometriotic cysts, a diagnosis of endometriosis is made in the absence of endometrial-type stroma. We acknowledge that, in such instances, CD10 staining is of no value in confirming a diagnosis of endometriosis. In fact, many of these endometriotic cysts are probably benign neoplasms and are not related to usual pelvic/abdominal endometriosis. Studies using X linked polymorphic markers have demonstrated that ovarian endometriotic cysts may be monoclonal, ${ }^{67}$ supporting the hypothesis of a benign neoplasm.

"Endometriosis is defined as the presence of ectopic non-neoplastic endometrial tissue, usually glands and stroma, outside the uterus"

In summary, analogous to the situation within the uterus, CD10 is a sensitive immunohistochemical marker of endometrial stromal cells at ectopic sites. In cases of suspected endometriosis where the reporting pathologist is unsure whether or not endometrial-type stroma is present, CD10 staining is of value in establishing a definitive diagnosis of endometriosis.

\section{Authors' affiliations \\ V P Sumathi, W G McCluggage, Department of Pathology, Royal Group of Hospitals Trust, Grosvenor Road, Belfast BT12 6BL, Northern} Ireland

Correspondence to: Dr W G McCluggage, Department of Pathology, Royal Group of Hospitals Trust, Grosvenor Road, Belfast BT12 6BL, Northern Ireland; glenn.mccluggage@bll.n-i.nhs.uk

Accepted for publication 5 November 2001

\section{REFERENCES}

1 Greaves MF, Brown G, Rapsan T, et al. Antisera to acute lympho-blastic leukaemia cells. Clin Immunol Immunopathol 1975:4:67-84.

2 Chu P, Arber DA. Paraffin section detection of CD 10 in 505 non-hematopoietic neoplasms. Frequent expression in renal cell carcinoma and endometrial stromal sarcoma. Am J Clin Pathol 2000;113:374-82.

3 McCluggage WG, Sumathi VP, Maxwell P. CD 10 is a sensitive and diagnostically useful immunohistochemical marker of normal endometrial stroma and of endometrial stromal neoplasms. Histopathology $2001 ; 39: 273-8$

4 Chu PG, Arber DA, Weiss LM, et al. Utility of CD10 in distinguishing between endometrial stromal sarcoma and uterine smooth muscle tumors: an immunohistochemical comparsion of 34 cases. Mod Pathol 2001;14:465-71.

5 Clement PB, Young RH, Scully RE. Stromal endometriosis of the uterine cervix. A variant of endometriosis that may simulate a sarcoma. Am J Surg Pathol 1991;14:449-55

6 Nilbert M, Pejovic T, Mandahi N, et al. Monoclonal origin of endometriotic cysts. Int J Gynecol Cancer 1995;5:61-3.

7 Yano T, Jimbo H, Yoshikawa H, et al. Molecular analysis of clonality in ovarian endometriotic cysts. Gynecol Obstet Invest 1999;47:41-5. 